Med Klin Intensivmed Notfmed 2021 · 116:138-145 https://doi.org/10.1007/s00063-020-00750-8 Eingegangen: 30 . Juli 2020

Überarbeitet: 8. September 2020

Angenommen: 16 . September 2020

Online publiziert: 28 . Oktober 2020

(c) Der/die Autor(en) 2020

\section{Redaktion}

M. Buerke, Siegen

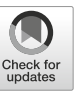

P. La Rosée $e^{1,2} \cdot$ H.-C. Bremer $\cdot$ F. La Rosée ${ }^{2,4} \cdot$ P. Mohm ${ }^{3} \cdot$ A. Hochhaus $^{5} \cdot$ I. Gehrke ${ }^{6}$. B. Kumle ${ }^{7} \cdot$ A. Benzing ${ }^{8} \cdot$ S. Russo $0^{8,9,10}$

' Klinik für Innere Medizin II, Hämatologie, Onkologie, Immunologie, Infektiologie und Palliativmedizin, Schwarzwald-Baar-Klinikum, Villingen-Schwenningen, Deutschland

${ }^{2}$ Medizinische Fakultät, Universitätsklinikum Jena, Friedrich-Schiller-Universität Jena, Jena, Deutschland ${ }^{3}$ Lungenzentrum, Schwarzwald-Baar-Klinikum, Donaueschingen, Deutschland

${ }^{4}$ Medizinische Fakultät Carl Gustav Carus, Technische Universität Dresden, Dresden, Deutschland

${ }^{5}$ Klinik für Innere Medizin II, Universitätsklinikum Jena, Jena, Deutschland

${ }^{6}$ Klinik für Innere Medizin IV, Innere Medizin Altersmedizin, Schwarzwald-Baar-Klinikum, Donaueschingen, Deutschland

${ }^{7}$ Klinik für Akut- und Notfallmedizin, Schwarzwald-Baar-Klinikum, Villingen-Schwenningen, Deutschland

${ }^{8}$ Klinik für Anästhesiologie, Intensiv-, Notfall- und Schmerzmedizin, Schwarzwald-Baar-Klinikum,

Villingen-Schwenningen \& Donaueschingen, Deutschland

${ }^{9}$ Medizinische Fakultät, Universität Göttingen, Göttingen, Deutschland

${ }^{10}$ Fakultät für Gesundheit, Universität Witten/Herdecke, Witten, Deutschland

\title{
Interdisziplinäres COVID-Board bei SARS-CoV-2-getriggerter hyperferritinämischer Inflammation
}

\section{Hintergrund und Fragestellung}

Eine Subgruppe der mit dem SARSCoV-2 Infizierten zeigt keine bis milde Symptome. Etwa $14 \%$ entwickeln schwere Krankheitszeichen mit stationärer Behandlungspflichtigkeit, weitere $5 \%$ benötigen eine intensivmedizinische Behandlung. Die intensivmedizinische Mortalität erreicht $50 \%$ [3]. Intensivmedizinische Aufnahmeindikation ist in der Regel die respiratorische Insuffizienz mit der Ausbildung eines akuten respiratorischen Distress-Syndroms (ARDS) auf dem Boden einer viralen Pneumonie.

Frühe Fallserien aus China wiesen bereits auf eine hyperferritinämische Inflammation im Sinne einer Zytokinsturmerkrankung als möglichen Mortalitätsfaktor hin [13, 23]. Erste Hypothesenpapiere zur Pathophysiologie des Multiorganversagens machten Anfang März 2020 auf ein aberrantes Immungeschehen aufmerksam, vergleichbar

P. Graf La Rosée und H.-C. Bremer haben den Beitrag zu gleichen Teilen verfasst. mit dem Makrophagen-AktivierungsSyndrom bzw. der MAS-ähnlichen Sepsis $[10,20]$. Vorab wurden mittlerweile voll publizierte Behandlungsserien mit der antirheumatischen Substanz, dem Interleukin-6-Rezeptor Antikörper Tocilizumab, kommuniziert [26]. Da auch Sektionsbefunde und ein Tiermodell die Rolle der die Lunge infiltrierenden Lymphozyten und Makrophagen mit peripherer Lymphozytenaktivierung als immunogenen Pathomechanismus des Organversagens bestätigten, wurde in China der zeitlich begrenzte Einsatz von Kortikosteroiden propagiert [25]. Bereits durch das erste SARS-verursachende Coronavirus (SARS-CoV) lagen tierexperimentelle Befunde zum pulmonal getriggerten Zytokinsturm und zur medikamentösen Inflammationshemmung vor [7]. Deutsche intensivmedizinische und pneumologische Fachgesellschaften waren bis zur Publikation der RecoveryStudie zurückhaltend bezüglich einer möglichen Immunmodulation, da auf das Potenzial der verzögerten VirusClearance und Begünstigung sekun- därer (Pilz-)Infektionen hingewiesen wurde [12, 14].

Vor dem Hintergrund einer fehlenden Standardtherapie bei lebensbedrohlicher COVID-19-Erkrankung wurden weltweit Patienten mit individuell indizierter Immunmodulation durch selektiv Zytokin-gerichtete Substanzen (Tocilizumab, Anakinra) oder Kinaseinhibitoren des Zytokin-Signalweges JAK/STAT (Ruxolitinib, Baricitinib) zur Dämpfung der Inflammation behandelt $[5,6,9,17,26]$.

Am Schwarzwald-Baar-Klinikum wurde am 04.03.2020 die erste SARSCoV-2-infizierte Patientin aufgenommen und parallel ein COVID-Behandlungsalgorithmus definiert. Zentrale Säulen der Qualitätsinitiative war die strikte räumliche Trennung durch ein ausschließlich COVID-19-Patienten aufnehmenden Zweit-Standort (Lungenzentrum Donaueschingen) und die Etablierung einer standortübergreifenden, interdisziplinären COVID-19-Videokonferenz („COVID-Board“). Die intensivmedizinische Fallkonferenz wird als Modell zur Identifikation und inter- 


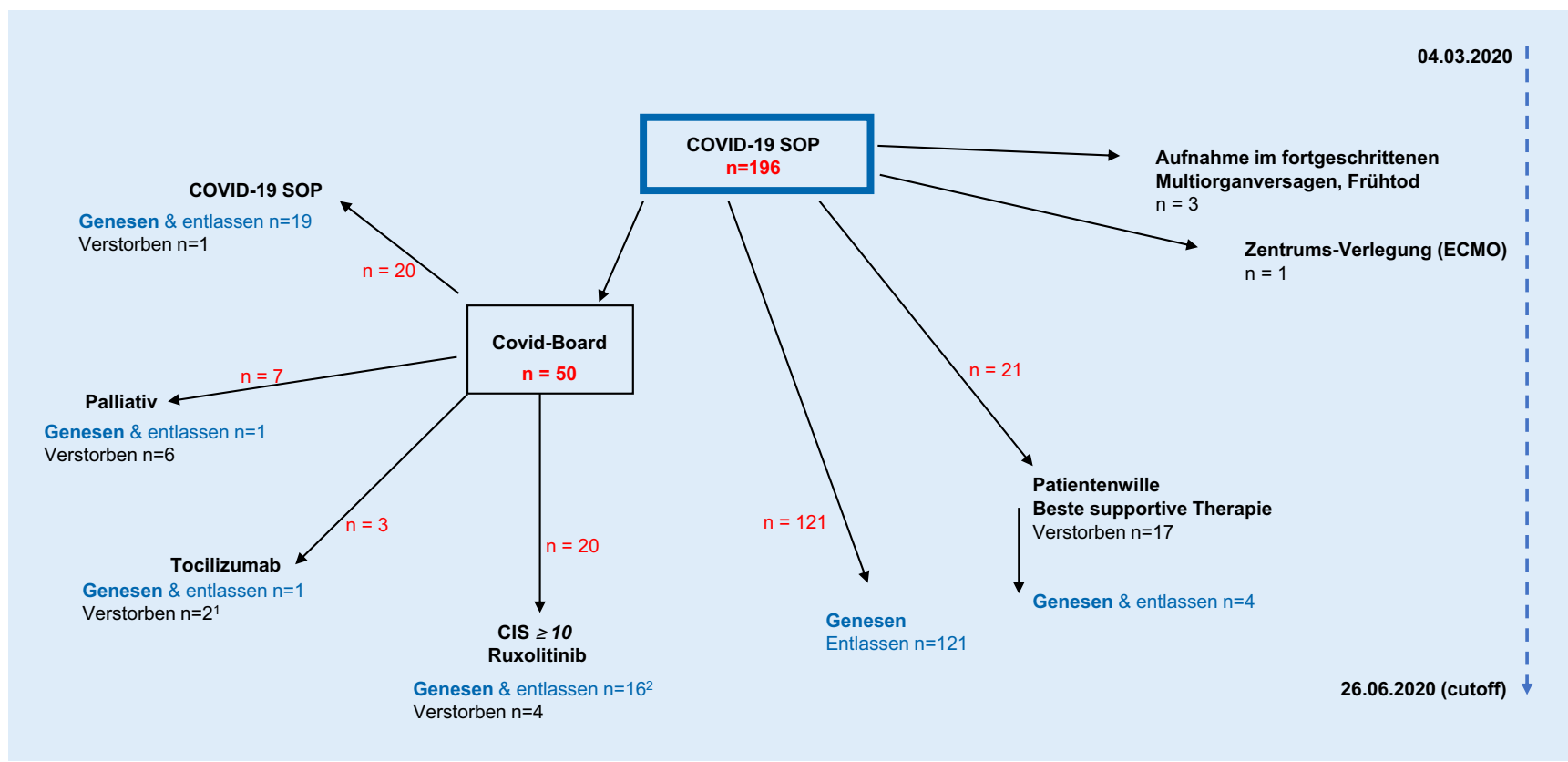

Abb. 1 A Consort-Diagramm der COVID-19-Patienten am Schwarzwald-Baar-Klinikum. 'Patient erhielt bei MAS-ähnlicher Sepsis Anakinra; ${ }^{2}$ ein Patient erhielt Tocilizumab vor Ruxolitinib

disziplinären Therapieführung seltener hyperferritinämischer Verlaufsformen sepsisähnlicher Krankheitsbilder vorgestellt.

\section{Studiendesign und Unter- suchungsmethoden}

\section{Krankenhausweite COVID-19-SOP}

Eine stadienabhängige medikamentöse Therapie mit hochdosiertem Vitamin C (1000 mg), Acetylsalicylsäure (500 mg), D-Dimer-stratifizierter Antikoagulation, Hydroxychloroquin (600 mg bid Tag 1, $200 \mathrm{mg}$ bid Tag 2-5) und Prednisolon (2 mg/kg Tag 1-3) wurde implementiert. Eine standardisierte COVID-19-Labordiagnostik wurde jeweils bei klinischer Verschlechterung zur Charakterisierung der Inflammation und Organschädigung veranlasst (-Tab. 1). Diese enthielt neben den üblichen Routineparametern Serumspiegel für Interleukin-6, den löslichen IL2-Rezeptor (sIL2R), Procalcitonin, Laktatdehydrogenase (LDH), Fibrinogen, D-Dimer, C-reaktives Protein (CRP) und ein Differenzialblutbild. Bezüglich der apparativen, beatmungsmedizinischen Differenzialtherapie wurden Patienten bis zu einem positiven endexpiratorischen Druck (PEEP) von $12 \mathrm{cmH}_{2} 0$, einem $\mathrm{p}_{\mathrm{a}} \mathrm{O}_{2} / \mathrm{F}_{\mathrm{i}} \mathrm{O}_{2}<90 \mathrm{~mm} \mathrm{Hg}$, inklusive Bauchlage, nichtinvasiv ventiliert (continuous positive airway pressure (CPAP) oder NIV) und auf Intermediate Care Station (IMC) behandelt, sofern dies von den Patienten toleriert wurde und nicht eine sofortige Intubation angezeigt war. Die Verlegungskriterien auf die Intensivstation (ITS) war neben einer katecholaminpflichtigen, hämodynamischen Instabilität vor allem eine fortgeschrittene intubationspflichtige respiratorische Insuffizienz.

\section{Therapiestratifikation nach Inflammationsgrad}

Zur Risikostratifikation wurde ein COVID-Inflammations-Score (CIS) auf dem Boden der zu diesem Zeitpunkt publizierten chinesischen COVID-Serien entwickelt (• Tab. 1; [17]). Patienten mit einem CIS $\geq 10$ (von 16 Punkten) wurden als hyperinflammatorisch eingestuft und in die interdisziplinäre, intensivmedizinische Fallkonferenz (COVID-Board) zur Indikationsprüfung möglicher immunmodulatorischer Therapieansätze eingeschlossen (• Abb. 1). Voraussetzung hierfür war die Abwesenheit einer unkontrollierten sekundären Infektion, das Fehlen terminaler Organinsuffizi- enz (vor SARS-CoV-2-Infektion) und eine Multimorbidität mit einer COVID19 unabhängigen Lebenszeitprognose $<6$ Monate.

\section{COVID-Board}

Eine tägliche telemedizinische Konferenzschaltung zwischen Zentralklinikum und Standort Donaueschingen der Fachdisziplinen Anästhesiologie, Pneumologie, Geriatrie/Palliativmedizin, $\mathrm{He}$ patologie/Allgemeine Innere Medizin und Hämatologie/Immunologie identifizierte hyperinflammatorische Patienten und erarbeitete eine dokumentierte Therapieempfehlung.

\section{Datenmanagement}

Die Datenanalyse basierte auf den Patientenakten und dem Klinikinformationssystem Orbis (AGFA Health Care GmbH, Bonn, Deutschland). Die anonymisierte Datenaufbereitung erfolgte in MS Excel (Microsoft, Redmond, WA, USA).

\section{Ethik}

Die anonymisierte, retrospektive Analyse erfolgte auf Grundlage eines Ethikvotums der Landesärztekammer BadenWürttemberg (Nr. F-2020-052). Die Un- 
tersuchung erfolgte gemäß der Deklaration von Helsinki.

\section{Ergebnisse}

\section{Patientencharakteristika}

Von 23.02.2020 bis 26.06.2020 wurden 196 Patienten mit symptomatischer COVID-19-Erkrankung am Schwarzwald-Baar-Klinikum behandelt (• Tab. 2). Hiervon wurden bei klinischer Verschlechterung bzw. bei primär kritischer Erkrankung $n=50$ Patienten im COVID-Board vorgestellt. Während das mediane Alter in der Gesamtkohorte (70 Jahre) nahezu dem in der Fallkonferenz entsprach (69 Jahre), wurden in der Fallkonferenz mehr männliche $\mathrm{Pa}$ tienten vorgestellt $(n=33 ; 66 \%)$. Zwei Drittel der Patienten litten in beiden Kohorten unter vorbekannten HerzKreislauf-Erkrankungen (66\%, 70\%). Diabetes mellitus fand sich in den COVID-Board-Patienten angereichert ( $29 \%$ vs. $38 \%)$. Onkologische Erkrankungen betrafen $20 \%$ in der Gesamtkohorte und $26 \%$ im COVID-Board.

\section{Inflammatorische Charakterisie- rung der Patienten im COVID- Board}

Zur Erfassung der hyperferritinämischen Inflammation wurden im COVID-Basislabor sowie bei klinischer Verschlechterung Parameter der Hyperinflammation (Differenzialblutbild, CRP, Triglyzeride, Ferritin, sIL2R, IL6, Procalcitonin) sowie der Hyperkoagulation (Quick, INR, PTT, Fibrinogen, D-Dimer) bestimmt. Radiologische Lungenbildgebung (konventionelles Röntgen-Thorax oder Computertomographie der Lunge) sowie Fieber wurden zusätzlich für den CIS einbezogen (•Tab. 2). Als Zeichen der hyperferritinämischen Inflammation und Korrelat der zentralen Rolle des Monozyten-Makrophagensystems der Lunge in der immunologischen COVID-Abwehr [21] fanden sich deutlich erhöhte Ferritinwerte bis maximal $3867 \mathrm{ng} / \mathrm{ml}$. Ergänzend zeigte als Surrogat der lymphozytären Aktivierung der sIL2R eine im Median 2-fache Erhöhung über den oberen Normwert

Med Klin Intensivmed Notfmed 2021 · 116:138-145 https://doi.org/10.1007/s00063-020-00750-8 ( ) Der/die Autor(en) 2020

P. La Rosée · H.-C. Bremer · F. La Rosée · P. Mohm · A. Hochhaus · I. Gehrke • B. Kumle · A. Benzing $\cdot$ S. Russo

\section{Interdisziplinäres COVID-Board bei SARS-CoV-2-getriggerter hyperferritinämischer Inflammation}

Zusammenfassung

Hintergrund. Patienten mit schwerer COVID-19-Erkrankung entwickeln eine hyperferritinämische Inflammation, ein sepsisähnliches Immundysregulationssyndrom. Methode. Retrospektive Kohortenanalyse nach Therapiestratifizierung in einer standortübergreifenden telemedizinischen Fallkonferenz. Frühzeitige, standardisierte Identifizierung von Patienten mit einem Risiko für einen schweren Verlauf (COVIDInflammation-Score; CIS) und Intubationsvermeidung mit Schwerpunkt auf nichtinvasive Ventilation (NIV) sind Kernelemente des Behandlungsalgorithmus. Patienten mit lebensbedrohlicher Inflammation wurde ein individueller Heilversuch mit dem Immunmodulator Ruxolitinib angeboten. Ergebnisse. Zwischen 04.03.2020 und 26.06.2020 wurden 196 COVID-19-Patienten behandelt. Der Altersmedian (70 Jahre) und die Komorbidität waren im Interstudienvergleich hoch. Die Gesamtmortalität lag bei $17,3 \%$, wobei bei der Hälfte der verstorbenen
Patienten eine A-priori-Therapielimitierung festgelegt war. Das CIS-Monitoring der mit Ruxolitinib behandelten Hochrisikopatienten $(n=20)$ ergab nach 5, 7 und 15 Tagen eine Inflammationssuppression um $42 \%$ (15-70), $54 \%$ (15-77) und $60 \%(15-80)$. In dieser Gruppe lag die Mortalität bei $20 \%$ (4/20). Die Gesamtmortalität adjustiert auf Patienten mit intendierter Maximaltherapie lag bei 8,7\% (17/196).

Schlussfolgerung. Die COVID-19-Pneumonie mit hyperferritinämischer Inflammation ist verwandt mit der MakrophagenAktivierungssyndrom-ähnlichen Sepsis. Eine interdisziplinäre Fallkonferenz als Qualitätsinstrument der Intensivmedizin zur Erfassung seltener sepsisähnlicher Krankheitsbilder wird vorgestellt.

\section{Schlüsselwörter}

Makrophagenaktivierungssyndrom-ähnliche Sepsis · Zytokinsturm · Ruxolitinib · Virale Pneumonie $\cdot$ Telemedizin

\section{Interdisciplinary COVID board for patients with SARS-CoV-2- triggered hyperferritinemic Inflammation}

\section{Abstract}

Background. Patients with severe COVID19 develop hyperferritinemic inflammation, a rare sepsis-like immune dysregulation syndrome.

Methods. Stratified treatment decisions in a cross-location telemedical interdisciplinary case conference were assessed in this retrospective cohort study. A standardized treatment algorithm including continuous positive airway pressure and noninvasive ventilation was implemented. A locally developed COVID inflammation score (CIS) defined patients at risk for severe disease. Patients with life-threatening inflammation were offered off-label treatment with the immune modulator ruxolitinib.

Results. Between 4 March 2020 and 26 June 2020 COVID-19 patients $(n=196)$ were treated. Median patient age ( 70 years) and comorbidity were high in interstudy comparison. Mortality in all patients was $17.3 \%$. However, advance care planning statements and physician directives limited treatment intensity in $50 \%$ of the deceased patients. CIS monitoring of ruxolitinib-treated high-risk patients $(n=20)$ on days 5,7 , and 15 resulted in suppression of inflammation by $42 \%(15-70), 54 \%(15-77)$ and $60 \%(15-80)$. Here, mortality was $20 \%(4 / 20)$. Adjusted for patients with a maximum care directive including ICU, total mortality was $8.7 \%$ (17/196).

Conclusion. Severe COVID-19 pneumonia with hyperferritinemic inflammation is related to macrophage activation syndromelike sepsis. An interdisciplinary intensive care teleconference as a quality tool for ICUs is proposed to detect patients with rare sepsislike syndromes.

\section{Keywords}

Macrophage activation syndrome like sepsis · Cytokine storm · Ruxolitinib · Viral pneumonia $\cdot$ Telemedicine 
Tab. 1 COVID-19-Inflammations-Score

(CIS; $\geq 10$ von 16). (Nach [17])

\begin{tabular}{ll}
\hline Kriterien & Punkte \\
\hline Radiologisch bds. Lungeninfiltrate & $\mathbf{3}$ \\
CRP $>20^{*}$ ULN & $\mathbf{2}$ \\
\hline Ferritin $>2 *$ ULN & $\mathbf{2}$ \\
\hline Triglyzeride $>1,5 *$ ULN & $\mathbf{1}$ \\
\hline IL-6 $>3{ }^{*}$ ULN & $\mathbf{1}$ \\
\hline Fibrinogen $>$ ULN & $\mathbf{1}$ \\
\hline Leukozyten $>$ ULN & $\mathbf{1}$ \\
\hline Lymphopenie $<1,1 / n L$ & 2 \\
Fieber $>38,5^{\circ} \mathrm{C}$ & $\mathbf{2}$ \\
Gerinnungsaktivierung & $\mathbf{1}$ \\
DIC (D-Dimer $>$ ULN) & \\
PTT $>$ ULN & \\
ULN Oberer Normalwert & \\
\hline
\end{tabular}

(1519 U/ml (285-7192)). Beide, Ferritin und sIL2R, lagen damit deutlich unterhalb der für die hämophagozytische Lymphohistiozytose oder das Makrophagen-Aktivierungssyndrom bekannt exzessiv erhöhten Werte [4], reihen sich jedoch gut in hyperferritinämische Inflammation bei Sepsispatienten ein [18].

\section{Mortalität und Komorbidität}

Die Gesamtkohorte zeigte eine Mortalität von $17,3 \%(34 / 196)$ und eine ITSMortalität von 45,2\% (14/31). Siebzehn der 21 Patienten, die a priori eine beste supportive Therapie ohne intensivmedizinische Therapieeskalation wünschten, sind unter palliativmedizinischer Therapie verstorben ( $\bullet$ Abb. 1). Damit ist die um diese Patienten bereinigte Mortalität 17/196 (8,7\%). Bei 7 der 50 in das COVID-Board eingebrachten Patienten wurde eine Therapielimitierung konsentiert, hiervon sind 6 Patienten verstorben, einer wurde genesen entlassen. Drei Patienten wurden im fortgeschrittenen Multiorganversagen stationär zugewiesen und sind innerhalb von Stunden in der ZNA $(n=1)$ oder auf Intensivstation $(n=2)$ verstorben. Eine Patientin wurde zur extrakorporalen Oxygenierung (ECMO) in ein universitäres Zentrum verlegt und ist dort verstorben. Die intensivmedizinische Gesamtletalität von 45,2 \% errechnet sich somit aus $\mathrm{Pa}$ tienten mit bereits bei Verlegung fortgeschrittenem respiratorischem bzw. Mul- tiorganversagen. Bei intubationsvermeidendem Beatmungsalgorithmus wurden NIV/CPAP-Patienten der IMC nicht der ITS-Grundgesamtheit zugeordnet.

\section{Immunmodulatorische Therapie}

Bei nahezu der Hälfte der Patienten ( $n=20 ; 40 \%$ ) wurde im COVID-Board bei CIS-Werten $\geq 10$ (Median: 12; Range: 10-14) eine Empfehlung zur Immunmodulation mit dem oralen JAK1/2Inhibitor Ruxolitinib ausgesprochen und in der Folge nach Einholung des „informed consent“ auch umgesetzt. In die hier vorgestellte Analyse gehen die 14 Patienten der Erstpublikation zu Ruxolitinib bei SARS-CoV-2 ein [17]. Vier der 20 (20\%) hyperinflammatorischen Hochrisikopatienten sind verstorben, 16 sind als genesen entlassen worden. Das Monitoring der Inflammation anhand klinischer Faktoren (Respiration, Fieber und des COVID-Labors) ergab nach 5, 7 und 15 Tagen eine CIS-Reduktion um $42 \%$ (15-70), 54\% (15-77) und 60\% (15-80) als Surrogat für die Inhibition der pathologischen Immunreaktion mit sepsisähnlichem Charakter.

\section{Diskussion}

Die COVID-19-Erkrankung führt bei einem Fünftel der SARS-CoV-2 positiv getesteten Patienten zu einem pulmonal getriggerten Krankheitsbild. Eine Subgruppe dieser Patienten entwickelt eine hyperferritinämische Inflammation mit drohendem Multiorganversagen.

\section{Hyperferritinämische} Inflammationssyndrome: COVID-19 und MAS-ähnliche Sepsis

Hyperferritinämische Inflammationssyndrome sind eine zunehmend bekannter werdende Gruppe intensivmedizinisch zu behandelnder Syndrome, zu denen u.a. die klassische Sepsis, die hämophagozytischen Syndrome HLH und MAS-HLH, die MAS-ähnliche Sepsis (MAS-like Sepsis) sowie die inflammatorischen Fiebersyndrome des konstitutionell aktivierten Inflammasoms (systemische juvenile idiopathi- sche Arthritis, Morbus Still, Cryopyrinassoziierte periodische Syndrome) und das iatrogene Zytokin-Release-Syndrom (CRS) gerechnet werden [8, 16]. Verschleppte Diagnosen seltener Inflammationssyndrome beruhen auf den häufig überlappenden Leitbefunden [20].

Für die hämophagozytische Lymphohistiozytose (HLH) konnte durch Analyse der Ferritinwerte aus einem großen Sepsiskollektiv retrospektiv eine $78 \%$ Fehldiagnoserate (7/9 Patienten) bei eigentlich vorliegender HLH eruiert werden [19]. Für die Subgruppe der MAS-ähnlichen Sepsis konnte post hoc aus einem großen, prospektiv mit dem Interleukin-1-Rezeptor-Antagonisten Anakinra behandelten Sepsiskollektiv ein $50 \%$ verbessertes Gesamtüberleben ausschließlich für MAS-like Patienten bei ansonsten negativem Studienergebnis für Anakinra berichtet werden [24]. Kürzlich wurden die positiven Ergebnisse der prospektiven britischen RECOVERY-Studie zu 10-tägiger niedrig dosierter Dexamethason-Therapie bei schwerer COVID-19-Erkrankung berichtet, welche eine Überlegenheit bzgl. Gesamtüberleben in der DexamethasonGruppe für $\mathrm{O}_{2}$ - und beatmungspflichtige Patienten zeigte [12].

Der von uns beschriebene Ansatz, über gezielte Inhibition des JAK/STATSignalweges mit Ruxolitinib den Zytokinsturm der COVID-19-Erkrankung molekular zu bremsen, zeigt einen weiteren vielversprechenden Ansatz der personalisierten Inflammationshemmung der COVID-19-Hyperferritinämie [5, 17]. Dabei ist die prospektive Risikostratifizierung mittels des neu entwickelten Score-Systems (CIS) hervorzuheben (- Tab. 1). Der CIS wird nun in einer prospektiven multizentrischen COVID-19-Therapiestudie (RuxCoFlam, NCT04338958) deutschlandweit validiert. Die in der vorgelegten Fallserie eruierte Gesamtmortalität der COVID19-Patienten von $17 \%$ bzw. $8,7 \%$ unter Ausschluss der a priori definierten Therapiezielbegrenzung, ist im Vergleich mit den international publizierten Zahlen vor dem Hintergrund hoher Interstudien-Variabilität bzgl. Eingangskriterien (Ausschluss Frühtod? Ausschluss von Patienten mit a priori Therapielimitation? 


\section{Originalien}

Tab. 2 Patienten-und Behandlungscharakteristika

\begin{tabular}{|c|c|c|c|}
\hline \multicolumn{2}{|l|}{ Charakteristika } & Gesamt $n=196$ & COVID-Board $n=50$ \\
\hline \multicolumn{2}{|l|}{ Alter, Jahre, Median (Range) - } & $70(0,1-95)$ & $69(38-85)$ \\
\hline \multicolumn{2}{|l|}{ Männlich, no. (\%) } & $104(53)$ & $33(66)$ \\
\hline \multicolumn{2}{|l|}{ Weiblich, no. (\%) } & $92(47)$ & $17(34)$ \\
\hline \multicolumn{4}{|l|}{ Komorbiditäten, no. (\%) } \\
\hline \multicolumn{2}{|l|}{ Herz-Kreislauf-Erkrankungen } & $130(66)$ & $35(70)$ \\
\hline \multicolumn{2}{|l|}{ Diabetes } & $56(29)$ & $19(38)$ \\
\hline \multicolumn{2}{|l|}{ Lungenerkrankung } & $45(23)$ & $12(24)$ \\
\hline \multicolumn{2}{|l|}{ Niereninsuffizienz } & $41(21)$ & $9(18)$ \\
\hline \multicolumn{2}{|l|}{ Maligne Erkrankung } & $39(20)$ & $13(26)$ \\
\hline \multicolumn{2}{|l|}{ Chronische NIV-Behandlung, no. (\%) } & $7(4)$ & $1(2)$ \\
\hline \multicolumn{4}{|l|}{ Behandlung, no. (\%) } \\
\hline \multicolumn{2}{|l|}{ Nur NIV- oder CPAP-Behandlung } & $46(23)$ & $24(48)$ \\
\hline \multicolumn{2}{|l|}{ Nur invasiv beatmet } & $8(4)$ & $3(6)$ \\
\hline \multicolumn{2}{|l|}{ NIV + invasive Beatmung } & $8(4)$ & $7(14)$ \\
\hline \multicolumn{2}{|l|}{ Beatmungstage, Median (Range) } & $9(1-68)$ & $11(1-68)$ \\
\hline \multicolumn{2}{|l|}{ ITS-Aufenhalt (\%) } & $31(16)$ & $19(38)$ \\
\hline \multicolumn{2}{|l|}{ ITS-Aufenhalt, Tage, Median (Range) - } & $10(1-63)$ & $12(4-63)$ \\
\hline \multicolumn{2}{|l|}{ Hydroxychloroquin (\%) } & $75(38)$ & $35(70)$ \\
\hline \multicolumn{2}{|l|}{ Kortikosteroide } & $36(18)$ & $27(54)$ \\
\hline \multicolumn{2}{|l|}{ Ruxolitinib } & $20(10)$ & $20(40)$ \\
\hline \multicolumn{2}{|l|}{ Tocilizumab } & $4(2)$ & $4(8)$ \\
\hline \multicolumn{2}{|l|}{ Temperatur Tag $1 \geq 38,5^{\circ} \mathrm{C}$, no. $(\%)^{\text {a }}$} & $21(11)$ & $8(16)$ \\
\hline Laborwerte Tag 1, median (Range) & \multicolumn{3}{|l|}{ Normwerte } \\
\hline Ferritin, ng/ml & $30-400$ & $490(6-3867)$ & $928(22-3867)$ \\
\hline \multicolumn{2}{|l|}{ IL-6, $x$ ULN $^{\mathrm{b}}$} & - & $10,88(0,2-1417,9)$ \\
\hline Maximaler sIL2r, $\mathrm{U} / \mathrm{ml}^{\mathrm{c}}$ & $158-623$ & - & $1519(285-7192)$ \\
\hline C-reaktives Protein, mg/l & $<5$ & $53,96(0-430)$ & $75,04(2,38-430)$ \\
\hline Procalcitonin, ng/ml & $<0,5$ & $0,07(0,01-203)$ & $0,11(0,01-3,54)$ \\
\hline D-Dimere, mg/l & $0,19-0,55$ & $1,095(0,1-186,6)$ & $1,32(0,1-22,17)$ \\
\hline Leukozyten, /nl & $4-10$ & $7,06(1,56-31,37)$ & $7,19(1,97-29,93)$ \\
\hline Lymphozyten absolut, /nl & $1,2-3,4$ & $1(0,07-4,31)$ & $0,9(0,07-2,38)$ \\
\hline \multicolumn{2}{|c|}{ Krankenhausverweildauer, Median (Range) - Tage } & $12(1-71)$ & $19(4-71)$ \\
\hline \multicolumn{2}{|l|}{ Mortalität (gesamt), no. (\%) } & $34(17,3)^{d}$ & $13(26)$ \\
\hline \multicolumn{2}{|c|}{ Verstorben nach a priori Therapielimitierung } & $17(50)$ & 0 \\
\hline Verstorben nach therapielimitierender $\mathrm{E}$ & im COVID-Board & - & $6(46)$ \\
\hline Mortalität (gesamt, ohne Pat. mit a prior & itierung), no. (\%) & $17(8,7)$ & - \\
\hline ITS-Mortalität, no. (\%) & & $14(45,2)^{d}$ & $9(47,4)$ \\
\hline $\begin{array}{l}\text { ITS Intensivstation, NIV nichtinvasive Beat } \\
\text { "Von } 186 \text { Patienten vorhanden } \\
\text { 'IL-6 in „upper level of normal", am Tag de } \\
\text { 'Maximaler sIL2R während des Krankenha } \\
\text { "Ein Patient nach Zentrumsverlegung (ECI } \\
\text { 'Ohne CPAP/NIV-beatmete IMC-Patienten }\end{array}$ & $\begin{array}{l}\text { ellung im COVID-BC } \\
\text { ner ITS verstorben }\end{array}$ & & \\
\hline
\end{tabular}

Versorgungsstruktur, Altersspektrum und Komorbiditäten) sehr vorsichtig einzuordnen [11, 22, 27].

\section{Fachärztliche Interdisziplinarität} auf Intensivstation

Die weltweit gesammelten Erfahrungen mit dem intensivmedizinischen Management von COVID-19 mit hy- perferritinämischer Inflammation weisen auf das potenzielle Risiko einer verpassten Immunmodulation bei sepsisähnlichen Krankheitsbildern. Hier berichten wir von einer interdisziplinären Fallbesprechung (COVID-Board) 


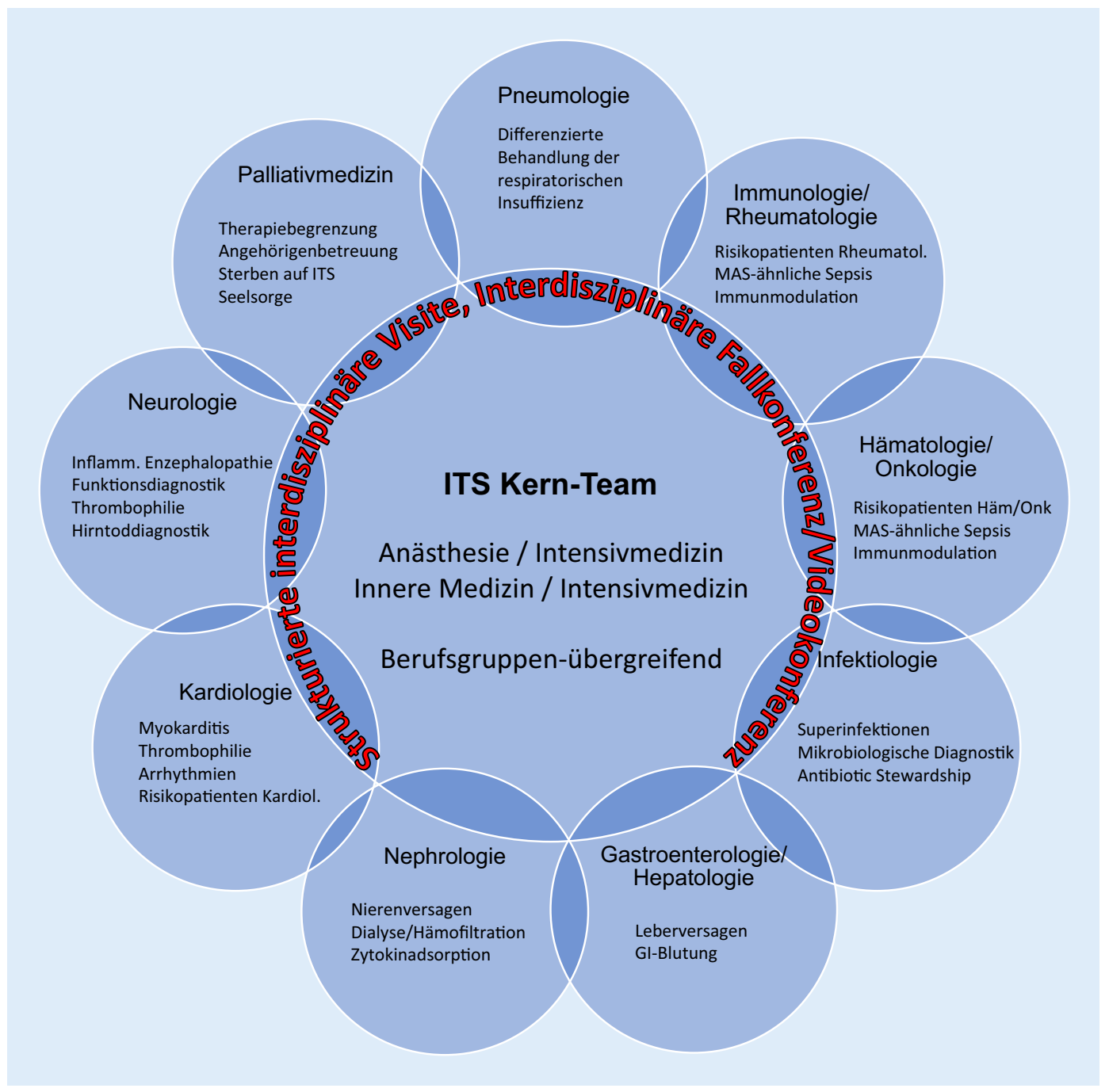

Abb. $2<$ COVID-Fallkonferenz, beteiligte Fachdisziplinen

unter kollegialer Leitung der Beatmungsmedizin (Pneumologie) und Intensivmedizin (Anästhesie) mit dem Ziel, gemeinsam die diagnostische Vigilanz zu erhöhen und die Differenzialtherapie der sepsisähnlichen Hyperinflammation durch gezielte Immunmodulation zu optimieren ( $\bullet$ Abb. 2). Hierfür zeigt die Literatur unseres Wissens noch keine Vorbildstrukturen. Im Wesentlichen konzentriert sich die Interdisziplinarität auf die Intensivtherapie und die kollegiale Zusammenarbeit zwischen den Berufsgruppen des Kernteams [1].

Unsere interdisziplinären Erfahrungen in der Behandlung der COVID-19Erkrankung unterstützen die Notwendigkeit einer fachärztlichen Interdisziplinarität bei ausgesuchten Patienten mit komplexen Krankheitsbildern. Gelebte Interdisziplinarität beruht in vielen
Bereichen derzeit noch auf engagierten Einzelpersönlichkeiten. Sie erscheint den Autoren jedoch unerlässlich für gegenseitigen Wissensgewinn, die ganzheitliche Betrachtung eines komplexen Krankheitsbildes und auch die Entwicklung entsprechender Studienkooperationen.

Einen beispielgebenden Lernprozess zur Interdisziplinarität stieß die Deutsche Krebsgesellschaft (DKG) durch das Zertifikat „Onkologisches Zentrum“ an. Interdisziplinarität wurde messbar und wirkte qualitätsverbessernd [15]. Wir werben daher für eine verbesserte Zusammenarbeit des intensivmedizinischen Kernteams mit Fachexpertise aus der Immunologie und Infektiologie. COVID-19 mit der aberranten, pulmonal getriggerten Immunantwort und der hyperferritinämischen Inflammation könnte hier als modellhafter Katalysator wirken. Die interdisziplinäre Fallkonferenz wäre mit eingebrachten Patienten als Kennzahl für das Peer Review ein geeignetes Instrument.

\section{Telemedizin in der Intensivmedizin}

COVID-19 wirkt auch als Katalysator für eine Kultivierung der klinischen Videokonferenzen, ein weiteres mögliches Qualitätsinstrument einer interdisziplinären Intensivmedizin, wie die vorgelegte Studie unseres Zwei-Standort-Klinikums zeigt. Telemedizin, als standortübergreifende Betreuung von Intensivpatienten, wird in der Literatur eher als Ausbildungsinstrument beschrieben, als eine Plattform für die fachärztlich interdisziplinäre Fallkonferenz [2]. Insbesondere für Häuser ohne ansässige Spezialdisziplinen (Onkolo- 
gen/Pneumologen/Infektiologen) wäre das telemedizinische Modell ein zu evaluierendes Qualitätsinstrument.

\section{Schlussfolgerung}

Mit der gezielten Inhibition des COVID19-Zytokinsturms durch den JAK1/2Inhibitor Ruxolitinib konnte in einem selektierten Patientengut eine effektive Inflammationskontrolle erreicht werden. Den komplexen, intensivmedizinischen Aspekten des COVID-19-Managements wurde in der vorgelegten monozentrischen Fallserie eines kommunalen Klinikums der Zentralversorgung durch telemedizinische, interdisziplinäre Fallbesprechung Rechnung getragen. Die Autoren schlagen vor, bei Patienten mit hyperferritinämischer Inflammation und Sepsisverdacht die immunologische und infektiologische Fachexpertise strukturell noch stärker in die klassische intensivmedizinische Therapie $\mathrm{zu}$ integrieren. Prospektive Untersuchungen sind zur Validierung der postulierten Qualitätsverbesserung erforderlich.

\section{Fazit für die Praxis}

- Die COVID-19-Pneumonie verursacht eine hyperferritinämische Hyperinflammation.

- Die hyperferritinämische Inflammation wurde mit einem neu entwickelten COVID-19-Inflammations-Score (CIS) charakterisiert.

- Die mit der MAS-ähnlichen Sepsis verwandte Hyperinflammation wurde bei fehlendem Therapiestandard als individueller Heilversuch mit dem JAK1/2-Tyrosinkinase-Inhibitor Ruxolitinib behandelt.

- Die interdisziplinäre Therapiefindung und das Monitoring der Patienten wurde standortübergreifend in einer telemedizinischen Fallkonferenz strukturiert.

- Die intensivmedizinische Entscheidungsfindung zur Erkennung und Behandlung seltener Krankheitsbilder bedarf den Instrumenten der strukturierten Interdisziplinarität.

\section{Korrespondenzadresse}

Prof. Dr. med. P. La Rosée

Klinik für Innere Medizin II, Hämatologie, Onkologie, Immunologie, Infektiologie und Palliativmedizin, Schwarzwald-Baar-Klinikum Klinikstr. 11, 78052 Villingen-Schwenningen, Deutschland

paul.larosee@sbk-vs.de

Funding. Open Access funding enabled and organized by Projekt DEAL.

\section{Einhaltung ethischer Richtlinien}

Interessenkonflikt. P. Graf La Rosée erhielt Honorare von Novartis Deutschland (AdBoard/Vorträge). A. Hochhaus erhielt für klinische Studien finanzielle Unterstützung von Novartis Deutschland. H.-C. Bremer, F. Graf La Rosée, P. Mohm, I. Gehrke, B. Kumle, A. Benzing und S. Russo geben an, dass kein Interessenkonflikt besteht.

Für diesen Beitrag wurden von den Autoren keine Studien an Menschen oder Tieren durchgeführt. Für die aufgeführten Studien gelten die jeweils dort angegebenen ethischen Richtlinien.

Open Access. Dieser Artikel wird unter der Creative Commons Namensnennung 4.0 International Lizenz veröffentlicht, welche die Nutzung, Vervielfältigung, Bearbeitung, Verbreitung und Wiedergabe in jeglichem Medium und Format erlaubt, sofern Sie den/die ursprünglichen Autor(en) und die Quelle ordnungsgemäßnennen, einen Link zur Creative Commons Lizenz beifügen und angeben, ob Änderungen vorgenommen wurden.

Die in diesem Artikel enthaltenen Bilder und sonstiges Drittmaterial unterliegen ebenfalls der genannten Creative Commons Lizenz, sofern sich aus der Abbildungslegende nichts anderes ergibt. Sofern das betreffende Material nicht unter der genannten Creative Commons Lizenz steht und die betreffende Handlung nicht nach gesetzlichen Vorschriften erlaubt ist, ist für die oben aufgeführten Weiterverwendungen des Materials die Einwilligung des jeweiligen Rechteinhabers einzuholen.

Weitere Details zur Lizenz entnehmen Sie bitte der Lizenzinformation auf http://creativecommons.org/ licenses/by/4.0/deed.de.

\section{Literatur}

1. Deutsche Interdisziplinäre Vereinigung für Intensiv- und Notfallmedizin (2017) Qualitätsindikatoren Intensivmedizin. https://www.divi. de/images/Dokumente/PeerReview/170610qualitaetssicherung-intensivmedizin-peerreview-qualitaetsindikatoren-liste-indikatoren2017-3-auflage.pdf.Zugegriffen:27. Juli 2020

2. Becker CD, Fusaro MV, Scurlock C (2019) Telemedicine in the ICU: clinical outcomes, economic aspects, and trainee education. Curr Opin Anaesthesiol 32:129-135

3. Bhatraju PK, Ghassemieh BJ, Nichols Met al (2020) Covid-19 in critically ill patients in the seattle region-case series. N Engl J Med. https://doi.org/ 10.1056/NEJMoa2004500

4. Birndt S, Schenk T, Heinevetter B et al (2020) Hemophagocytic lymphohistiocytosis in adults: collaborative analysis of 137 cases of a nationwide German registry. J Cancer Res Clin Oncol 146:1065-1077

5. Cao Y, Wei J, Zou L et al (2020) Ruxolitinib in treatment of severe coronavirus disease 2019 (COVID-19): a multicenter, single-blind, randomized controlled trial.J Allergy Clin Immunol. https://doi.org/10.1016/j.jaci.2020.05.019

6. Cavalli G, De Luca G, Campochiaro C et al (2020) Interleukin-1 blockade with high-dose anakinra in patients with COVID-19, acute respiratory distress syndrome, and hyperinflammation:a retrospective cohort study. Lancet Rheumatol 2:e325-e331

7. Channappanavar R, Fehr AR, Vijay R et al (2016) Dysregulated type I interferon and inflammatory monocyte-macrophage responses cause lethal pneumonia in SARS-CoV-infected mice. Cell Host Microbe 19:181-193

8. Colafrancesco S, Alessandri C, Conti F et al (2020) COVID-19 gone bad: a new character in the spectrum of the hyperferritinemic syndrome? Autoimmun Rev 19:102573

9. Dimopoulos G, De Mast Q, Markou N et al (2020) Favorable Anakinra responses in severe Covid19 patients with secondary hemophagocytic lymphohistiocytosis. Cell Host Microbe. https:// doi.org/10.1016/j.chom.2020.05.007

10. Giamarellos-Bourboulis EJ, Netea MG, Rovina N et al (2020) Complex immune dysregulation in COVID-19 patients with severe respiratory failure. Cell HostMicrobe. https://doi.org/10.1016/j.chom 2020.04.009

11. Grasselli G, Zangrillo A, Zanella A et al (2020) Baseline characteristics and outcomes of 1591 patients infected with SARS-CoV-2 admitted to ICUs of the Lombardy region, Italy. JAMA. https:// doi.org/10.1001/jama.2020.5394

12. Horby P, Lim WS, Emberson JR et al (2020) Dexamethasone in hospitalized patients with Covid-19-preliminary report. N Engl J Med. https://doi.org/10.1056/NEJMoa2021436

13. Huang C, Wang Y, Li X et al (2020) Clinical features of patients infected with 2019 novel coronavirus in Wuhan, China. Lancet 395:497-506

14. Kluge S, Janssens U, Welte T et al (2020) Recommendations for critically ill patients with COVID-19. Med Klin Intensivmed Notfmed 115:175-177

15. Kowalski C, Graeven U, Von Kalle C et al (2017) Shifting cancer care towards multidisciplinarity: the cancer center certification program of the German cancer society. BMC Cancer 17:850

16. Kumar V (2018) Inflammasomes: Pandora's box for sepsis. J Inflamm Res 11:477-502

17. La Rosee F, Bremer HC, Gehrke I et al (2020) The Janus kinase $1 / 2$ inhibitor ruxolitinib in COVID19 with severe systemic hyperinflammation. Leukemia. https://doi.org/10.1038/s41375-0200891-0

18. Lachmann G, Knaak C, Vorderwulbecke $G$ et al (2019) Hyperferritinemia in critically ill patients. Crit Care Med. https://doi.org/10.1097/CCM. 0000000000004131

19. Lachmann G, Spies C, Schenk T et al (2018) Hemophagocyticlymphohistiocytosis: potentially underdiagnosed in intensive care units. Shock 50:149-155

20. Mehta P, McAuley DF, Brown M et al (2020) COVID-19: consider cytokine storm syndromes and immunosuppression. Lancet 395:1033-1034 
21. Merad M, Martin JC (2020) Pathological inflammation in patients with COVID-19: a key role for monocytes and macrophages. Nat Rev Immunol 20:355-362

22. Rosenberg ES, Dufort EM, Udo T et al (2020) Association of treatment with hydroxychloroquine or azithromycin with in-hospital mortality in patients with COVID-19 in New York State. JAMA. https://doi.org/10.1001/jama.2020.8630

23. Ruan Q, Yang K, Wang W et al (2020) Clinical predictors of mortality due to COVID-19 based on an analysis of data of 150 patients from Wuhan, China. Intensive Care Med. https://doi.org/10. 1007/s00134-020-05991-x

24. Shakoory B, Carcillo JA, Chatham WW et al (2016) Interleukin-1 receptor blockade is associated with reduced mortality in sepsis patients with features of macrophage activation syndrome: reanalysis of a prior phase III trial. Crit Care Med 44:275-281

25. Shang L, Zhao J, Hu Y et al (2020) On the use of corticosteroids for 2019-nCoV pneumonia. Lancet 395:683-684

26. Xu X, Han M, Li T et al (2020) Effective treatment of severe COVID-19 patients with tocilizumab. Proc Natl Acad Sci USA 117:10970-10975

27. Zhou F, Yu T, Du R et al (2020) Clinical course and risk factors for mortality of adult inpatients with COVID-19 in Wuhan, China: a retrospective cohort study. Lancet 395:1054-1062

\section{Erratum}

Med Klin Intensivmed Notfmed 2021 · 116:145

https://doi.org/10.1007/s00063-020-00763-3

Online publiziert: 8. Dezember 2020

(c) Springer Medizin Verlag GmbH, ein Teil von Springer Nature 2020

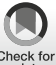

Check for
updates

P. La Rosée ${ }^{1,2} \cdot$ H.-C. Bremer ${ }^{3}$ F. La Rosée ${ }^{2,4} \cdot$ P. Mohm ${ }^{3} \cdot$ A. Hochhaus ${ }^{5}$. I. Gehrke ${ }^{6}$. B. Kumle ${ }^{7}$ A. Benzing ${ }^{8}$. S. Russo $0^{8,9,10}$

${ }^{1}$ Klinik für Innere Medizin II, Hämatologie, Onkologie, Immunologie, Infektiologie und Palliativmedizin, Schwarzwald-Baar-Klinikum, Villingen-Schwenningen, Deutschland

${ }^{2}$ Medizinische Fakultät, Universitätsklinikum Jena, Friedrich-Schiller-Universität Jena, Jena, Deutschland ${ }^{3}$ Lungenzentrum, Schwarzwald-Baar-Klinikum, Donaueschingen, Deutschland

${ }^{4}$ Medizinische Fakultät Carl Gustav Carus, Technische Universität Dresden, Dresden, Deutschland

${ }^{5}$ Klinik für Innere Medizin II, Universitätsklinikum Jena, Jena, Deutschland

${ }^{6}$ Klinik für Innere Medizin IV, Innere Medizin Altersmedizin, Schwarzwald-Baar-Klinikum, Donaueschingen, Deutschland

${ }^{7}$ Klinik für Akut- und Notfallmedizin, Schwarzwald-Baar-Klinikum, Villingen-Schwenningen, Deutschland ${ }^{8}$ Klinik für Anästhesiologie, Intensiv-, Notfall-und Schmerzmedizin, Schwarzwald-Baar-Klinikum, Villingen-Schwenningen \& Donaueschingen, Deutschland

${ }^{9}$ Medizinische Fakultät, Universität Göttingen, Göttingen, Deutschland

${ }^{10}$ Fakultät für Gesundheit, Universität Witten/Herdecke, Witten, Deutschland

\section{Erratum zu: Interdisziplinäres COVID-Board bei SARS-CoV-2- getriggerter Hyperferritin- ämischer Inflammation}

\section{Erratum zu:}

Med Klin Intensivmed Notfmed 2020

https://doi.org/10.1007/s00063-020-00

$750-8$

In der zunächst veröffentlichten OnlineVersion des Beitrags war der Name des Autors P. La Rosée falsch geschrieben. Bitte beachten Sie die korrigierte Schreibweise. Der ursprüngliche Artikel wurde korrigiert.

\section{Korrespondenzadresse}

Prof. Dr. med. P. La Rosée

Klinik für Innere Medizin II, Hämatologie, Onkologie, Immunologie, Infektiologie und Palliativmedizin, Schwarzwald-Baar-Klinikum Klinikstr. 11, 78052 Villingen-Schwenningen, Deutschland

paul.larosee@sbk-vs.de 